\title{
miR-34a Enhances the Susceptibility of Gastric Cancer to Platycodin D by Targeting Survivin
}

\author{
Yao Peng ${ }^{a}$ Jing-Ying Fan ${ }^{b}$ Jian Xiong ${ }^{c}$ Yu Lou ${ }^{c}$ Ying Zhu $^{d}$ \\ a Intensive Care Unit, Second Affiliated Hospital of Hunan University of Traditional Chinese Medicine, Changsha, PR \\ China; ${ }^{b}$ College of Integrated Chinese and Western Medicine, Hunan University of Traditional Chinese Medicine, \\ Changsha, PR China; ' Graduate School of Hunan University of Traditional Chinese Medicine, Changsha, PR China; \\ ${ }^{\mathrm{d}}$ Department of Gastroenterology, First Affiliated Hospital of Hunan University of Traditional Chinese Medicine, \\ Changsha, PR China
}

\section{Keywords}

miR-34a · Survivin · Platycodin D · Susceptibility · Gastric cancer

\begin{abstract}
Introduction: Platycodin D (PD), a triterpenoid saponin isolated from Platycodon grandiflorum, has a well-known antitumor effect in multiple human cancers, including gastric cancer (GC). miR-34a plays an important role in the progression of GC. However, the relationship between miR-34a and the susceptibility of GC cells to PD is still unclear. The aim of our research was to investigate the functions of miR-34a in mediating the susceptibility of GC to PD. Methods: qPCR was performed to detect the expression level of miR-34a and survivin in GC cells. The expression of survivin, $\mathrm{Bcl}-2$, Bax, and cleaved caspase-3 was analyzed using Western blot. Cell viability was detected by MTT assay, and apoptosis was analyzed via Annexin V-FITC/PI staining followed by flow cytometry. The colony formation and scratch-wound assays were applied to assess cell proliferation and migration. Caspase-3/7 activity was detected by a Caspase-Glo ${ }^{\circledR} 3 / 7$ detection kit. The relationship between miR-34a and survivin was determined by dual luciferase reporter gene assay. Fi-
\end{abstract}

nally, a GC xenograft mouse model was used to confirm our findings in vivo. Results: The expression of miR-34a decreased but survivin increased inversely in human GC cells. Survivin is a direct target of miR-34a and may be negatively regulated by miR-34a. PD could inhibit GC cell proliferation and induce apoptosis. Importantly, overexpression miR-34a or suppressing survivin was shown to enhance the susceptibility of GC to PD both in vitro and in vivo. Conclusions: miR34 a could modulate the susceptibility of GC to PD via targeting survivin, suggesting miR-34a overexpression may serve as a novel strategy to sensitize GC to anti-cancer drugs.

(c) 2019 S. Karger AG, Basel

\section{Introduction}

Gastric cancer (GC) is one of the most common malignant diseases in the digestive system [1], and is also the second leading cause of cancer-related death worldwide [2]. Due to the improvement of diet and the development of healthcare techniques, a steady decline in the incidence and mortality of GC has been observed in recent years [1, 3]. However, for late-stage GC patients, the treatment strategy is still limited and the prognosis is poor [2].

\section{KARGER}

(C) 2019 S. Karger AG, Basel 
Therefore, it is of great significance to explore effective agents to supplement current GC therapy to improve the survival of patients.

Platycodin D (PD), a triterpenoid saponin extracted from the roots of Platycodon grandiflorum [4], has been proven to possess anti-tumor effects on several malignancies, including breast cancer, hepatocellular carcinoma, and attenuated obesity in $\mathrm{db} / \mathrm{db}$ mice [5-8]. Kim and colleagues [9] recently reported that PD exerted strong tumor suppressive effects against GC through inhibiting proliferation and inducing apoptosis.

It has been widely recognized that microRNAs (miRNAs) play indispensable roles in tumorigenesis and disease progression $[10,11]$. For instance, Let- $7 b$ could decrease the drug resistance of GC cells through targeting AURKB [12]. miR-34a was found to be downregulated in GC tissues, and silencing miR-34a markedly inhibited apoptosis and DNA damage in GC cells $[13,14]$. Moreover, as a tumor suppressor, miR-34a could directly target HK1 and thus sensitize GC cells to luteolin [15]. Survivin, coded by BIRC5, a member of the inhibitor of apoptosis proteins family, has already become an anti-tumor therapeutic target. It directly inhibits the activity of apoptotic executional enzymes caspase- 3 and caspase-7 $[16,17]$. Survivin has been shown to be overexpressed in GC, and its knockdown significantly repressed cell proliferation and promoted apoptosis [18]. We have predicted that miR-34a could bind to the $3^{\prime}$ UTR of BIRC5 mRNA by bioinformatic analysis. So far, no study has reported that the interaction between miR-34a and survivin could affect the sensitivity of GC to natural anti-tumor compounds.

Here, we reveal that miR-34a was downregulated in GC, and miR-34a could target survivin and thus improve the sensitivity of GC to PD. Our data have provided a theoretical basis for the application PD for GC therapy and the feasibility of enhancing its efficacy.

\section{Materials and Methods}

\section{Cell Culture}

Human GC cell lines (NCI-N87, MKN-28, AGS, HGC-27, and SGC-7901) and the normal human gastric epithelial cell line (GES1) were obtained from the Cell Bank of Shanghai Institute of Cell Biology (Shanghai, China). Cell lines were cultured in RPMI-1640 medium supplemented with $10 \%$ fetal bovine serum (FBS, Gibco, USA) and $1 \%$ penicillin $(100 \mathrm{U} / \mathrm{mL}) /$ streptomycin $(100 \mathrm{U} / \mathrm{mL})$ at $37^{\circ} \mathrm{C}$ in an environment with $5 \% \mathrm{CO}_{2}$.

\section{Cells Transfection}

miR-34a mimics (sense: $5^{\prime}$-UGGCAGUGUCUIAGCUGGUUGU-3', antisense: 5' ${ }^{\prime}$-AACCAGCUAAGACACUGCCAUU-3'), corresponding control sequences (miR-NC, sense: $5^{\prime}$-UUCUCCGAACGUGUCACGUTT-3' , antisense: $5^{\prime}$-ACGUGACACGUUCGGAGAATT-3'), were synthesized by Genepharma (Shanghai, China), and the survivin-RNAi-LV and LV-RNAi were obtained from Genechem (Shanghai, China). According to manufacturer's instruction, plasmids and oligonucleotides were transfected into SGC-7901 cells after being cultured to $50-70 \%$ confluence using Lipofectamine 3000 (Invitrogen, USA). Cells transfected for $48 \mathrm{~h}$ were used for subsequent experiments.

\section{Cell Apoptosis Assay by Flow Cytometry}

After the treatment as previously described, all cells were harvested by trypsin and then washed with cold PBS three times. Cells were resuspended in $200 \mu \mathrm{L}$ of binding buffer. According to the Apoptosis Detection Kit (Sigma, USA) manufacturer's suggestion, cells were stained with propidium iodide (PI) and Annexin VFITC in a dark room for $15 \mathrm{~min}$. Then, the apoptosis cells were detected with a flow cytometer (BD Biosciences, San Jose, CA, USA) and analyzed by FlowJo software (LLC, USA).

MTT Assay

Cell proliferation was determined by MTT assay. Briefly, cells were seeded at a density of $5 \times 10^{3}$ cells per well in 96-well plates and treated with PD, miR-34a mimics, and miR-NC or survivinRNAi-LV and LV-RNAi. After culture, all cells were cultured with medium containing $20 \mu \mathrm{L}$ of MTT solution (Sigma) for $4 \mathrm{~h}$. Then, the cells were treated with DMSO for 10 min. Finally, an automatic microplate reader (BioTek, Vermont, USA) was used to detect the absorbance at $490 \mathrm{~nm}$ in wells.

\section{RNA Extraction and $q P C R$}

According to the manufacturer's instructions, total RNA was extracted from cells using an E.Z.N.A. ${ }^{\circledR}$ Total RNA Kit II (Omega, USA), and then transcribed into cDNA using a Primscript RT Reagent Kit (Takara, Japan). Then, the expression levels of miR-34a and survivin in different groups were determined by SYBR $^{\circledR}$ Green Premix Ex Taq ${ }^{\mathrm{TM}}$ (Takara, Japan). Finally, qPCR was performed in an Applied Biosystems 7500 system (Applied Biosystems, USA). The expression level of U6 snRNA and GAPDH were the determined internal references for miR-34a and survivin, respectively. Relative RNA levels were calculated as the fold changes with the $2^{-\Delta \Delta C T}$ formula. The specific primers used were as follows: hsa-miR-34a forward: $5^{\prime}$-CCGTGGCAGTGTCTTAGCT-3'; hsa-miR-34a reverse: $5^{\prime}$-CGGCCCAGTGTTCAGACTAC-3'; U6 forward: 5'-CTCGCTTCGGCAGCACA-3'; U6 reverse: $5^{\prime}$-AACGCTTCACGAATTTGCGT-3'; survivin forward: $5^{\prime}$-TGACGACCCCATGCAAAGGA-3'; survivin reverse: 5'-TAAACCCTGGAAGTGGTGCAG-3'; GAPDH forward: 5'-CCAGGTGGTCTCCTCTGA-3'; GAPDH reverse: 5'-GCTGTAGCCAAATCGTTGT-3'.

\section{Western Blotting}

The cells were harvested after treatment as previously described, followed by lysis using RIPA lysate (Beyotime Biotech, China). The protein concentration was then quantified using a BCA Protein Assay Kit (Beyotime Biotech) according to manufacturer's instructions. Then, the samples were separated using $10 \%$ SDS-PAGE gel and transferred onto PVDF membranes via electroporation, followed by blocking with $5 \%$ low-fat milk for $1 \mathrm{~h}$. After washing three times with PBS, the membranes were incu- 


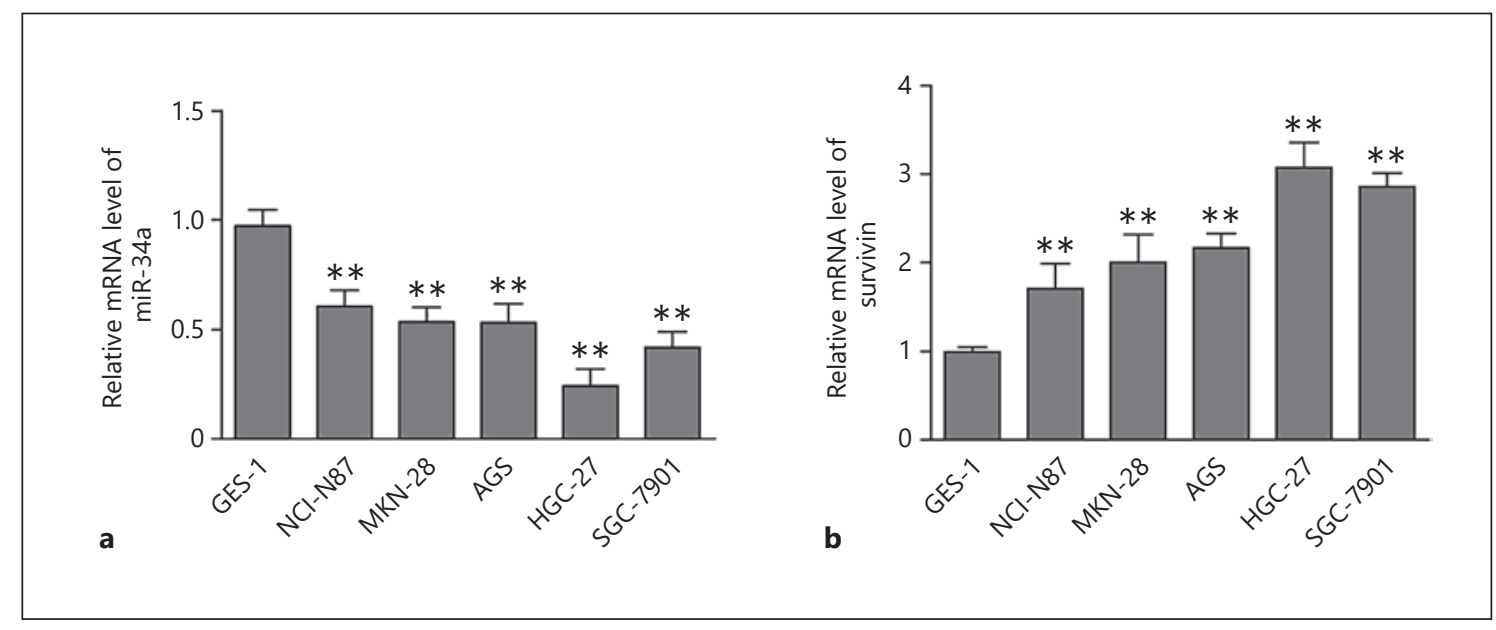

Fig. 1. Expression of miR-34a and survivin in GC cells. qPCR analysis of the expression of miR-34a (a) and survivin (b) increased in GC cell lines (NCI-N87, MKN-28, AGS, HGC-27, and SGC-7901) and the normalized gastric epithelial cell line (GES-1). ${ }^{* *} p<0.01$ versus GES-1.

bated with primary antibodies overnight at $4{ }^{\circ} \mathrm{C}$. After washing with TBST, membranes were incubated with secondary antibodies at $37^{\circ} \mathrm{C}$ for $1 \mathrm{~h}$. Finally, the bands were detected by ECL solution using a Bio Doc It system (Bio-Rad, USA), and quantified by Quantity One software. In this study, all primary antibodies were purchased from Abcam (Cambridge, USA), including survivin $(1: 1,000), \operatorname{Bcl}-2(1: 1,000), \operatorname{Bax}(1: 1,000)$, and cleaved caspase-3 $(1: 1,000)$. The secondary antibody $(1: 5,000)$ was purchased from Sigma (USA). The expression of GAPDH $(1: 3,000)$ was considered as the internal reference for correction.

Dual Luciferase Reporter Gene Assay

Dual luciferase reporter gene assays were used to investigate the interaction between miR-34a and the $3^{\prime} \mathrm{UTR}$ of mRNA produced by BIRC5. The human GC cells, SGC-7901, were seeded on 24-well plates, and cotransfected with $300 \mathrm{ng} /$ well of dual luciferase vectors for wild-type BIRC5 (BIRC5-WT) or mutated BIRC5 (BIRC5MUT) plasmid and $30 \mathrm{ng} /$ well miR-34a mimics or miR-NC vector (Applied Biosystems) using Lipofectamine 2000 (Invitrogen). Then, cells were incubated at $37^{\circ} \mathrm{C}$ for $48 \mathrm{~h}$. According to the manufacturer's suggestions, cells were harvested and lysed, and then assessed with a Dual-Luciferase Reporter Assay System (Promega, Fitchburg, WI, USA).

\section{Detection of Caspase-3/7 Activity Test}

The SGC-7901 cells were seeded at a density of $1 \times 10^{4}$ cells per well in 96-well plates, then the cells were treated with PD and/or miR-34a. After $48 \mathrm{~h}$ of treatment, the caspase-3/7 activity of the cells was determined by Caspase-Glo ${ }^{\circledR} 3 / 7$ detection kit (Promega) according to the manufacture's protocol.

\section{Soft Agar Colony Formation Assay}

To check if survivin and PD were involved in human GC cells, soft agar colony formation assay was performed in this research. SGC-7901 cells were treated with PD and LV-RNAi or survivinRNAi-LV. After $48 \mathrm{~h}$, cells were seeded in a 6-well plate precoated with agar at a density of 10,000 cells per well, and then incubated for 10 days. A microscope was used to capture the images of colonies in each well, and then analyzed using Image J.

\section{Wound Healing Assay}

Cell migration ability was examined by the wound healing assay. Briefly, after being treated with PD and LV-RNAi or survivinRNAi-LV, SGC-7901 cells were cultured in 6-well plates for $24 \mathrm{~h}$ and then scratched with a $200-\mu \mathrm{L}$ pipette tip. After washing with PBS, the non-adherent cells were removed, and the scratches were captured using the microscope. The cells were then incubated at $37^{\circ} \mathrm{C}$ for $48 \mathrm{~h}$, followed by capture again. Finally, five random nonoverlapping images in each well were selected and the migration distances of SGC-7901 cells was analyzed with the following formula: $\left(\mathrm{S}_{0 \mathrm{~h}}-\mathrm{S}_{48 \mathrm{~h}}\right) / \mathrm{S}_{0 \mathrm{~h}} \times 100 \%$.

\section{Tumor Growth in Nude Mice}

Overexpressing miR-34a (miR-34a-LV) and suppressing survivin (survivin-RNAi-LV) were obtained from Genechem (Shanghai, China). Xenograft tumors were generated via the subcutaneous injection of stable transfected cells $\left(5 \times 10^{6}\right)$ into nude mice $(\mathrm{BALB} / \mathrm{c}, 20 \pm 2 \mathrm{~g})$. The animals were treated with PD twice a week, and the tumor size was measured every 3 days. At 28 days postinjection, the mice were sacrificed for tumor photography and weight examination.

\section{Statistical Analysis}

In this study, all data were analyzed using SPSS 23.0 statistical software (SPSS, USA) and statistical differences between two experiments groups were analyzed by the Student $t$ test. Multiple group comparisons were made and analyzed with one-way analysis of variance. All data are presented as the mean \pm SD. Furthermore, $p$ values $<0.05$ were considered statistically significant. 


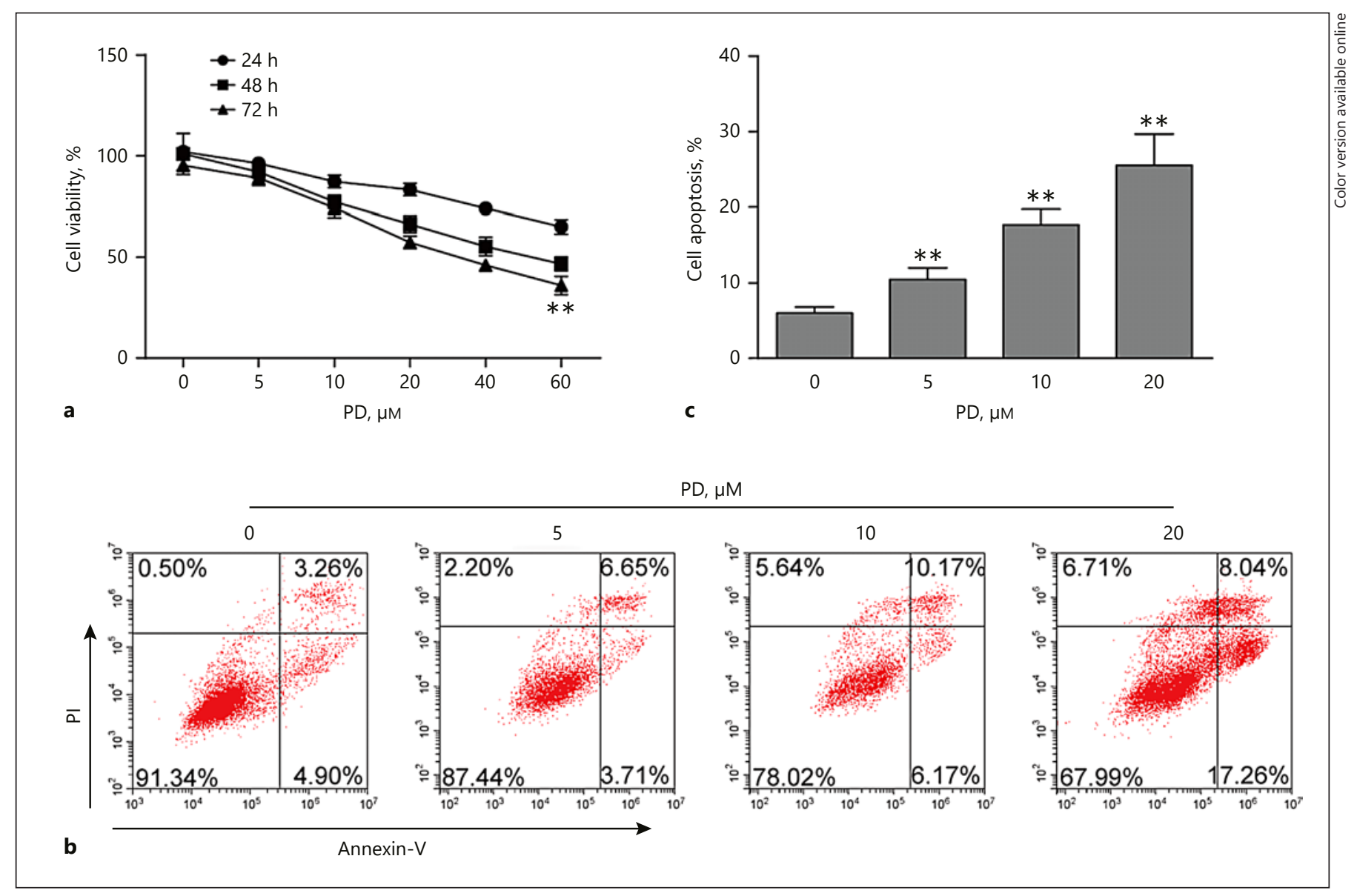

Fig. 2. PD inhibited the proliferation and promoted the apoptosis in GC cells. a SGC-7901 cells were treated with PD $(0-60 \mu \mathrm{M})$ for 24,48 , and $72 \mathrm{~h}$, then measured at $490 \mathrm{~nm}$ after adding the MTT reagent. $* * p<0.01$ versus the control. b, $\mathbf{c}$ The apoptosis rate of SGC-7901 cells was calculated after being treated with PD $(5,10,20 \mu \mathrm{M})$ for $48 \mathrm{~h}$ by flow cytometry. ${ }^{* *} p<0.01$ versus the control $(0 \mu \mathrm{M})$.

\section{Results}

The Expression of miR-34a and Survivin in GC Cells qPCR was performed to detect the expression levels of miR-34a and survivin in GC cells (NCI-N87, MKN-28, AGS, HGC-27, SGC-7901) and normal gastric epithelial cells (GES-1), respectively. As shown in Figure 1, the expression of miR-34a was significantly reduced in GC cells compared with the normal gastric epithelial cell line GES-1, while survivin expression was increased in GC cells. These results indicate that miR-34a expression was negatively correlated with the expression of survivin in the GC cells.

\section{PD Inhibits the Proliferation and Promotes the}

Apoptosis in GC Cells

We performed MTT assay and flow cytometry to detect the impact of PD on the proliferation and apoptosis in GC cells. As shown in Figure 2a, the MTT results showed that PD significantly inhibited the proliferation of GC cells in dose- and time-dependent manners, suggesting a cytotoxic effect of PD on GC cells. Furthermore, flow cytometric analysis demonstrated that $\mathrm{PD}$ promoted the apoptosis in GC cells (Fig. 2b, c). These results indicated that PD dose-dependently suppressed the proliferation and promoted the apoptosis of GC cells.

\section{miR-34a Inhibits the Expression of Survivin}

First, we assessed the effects of PD on the expression of miR-34a and survivin. As shown in Figure 3a, qPCR results suggested that $\mathrm{PD}$ promoted the expression of miR-34a, while it significantly suppressed the expression of survivin. Additionally, Western blotting showed that PD inhibited the expression of the protein level of survivin (Fig. 3b). 

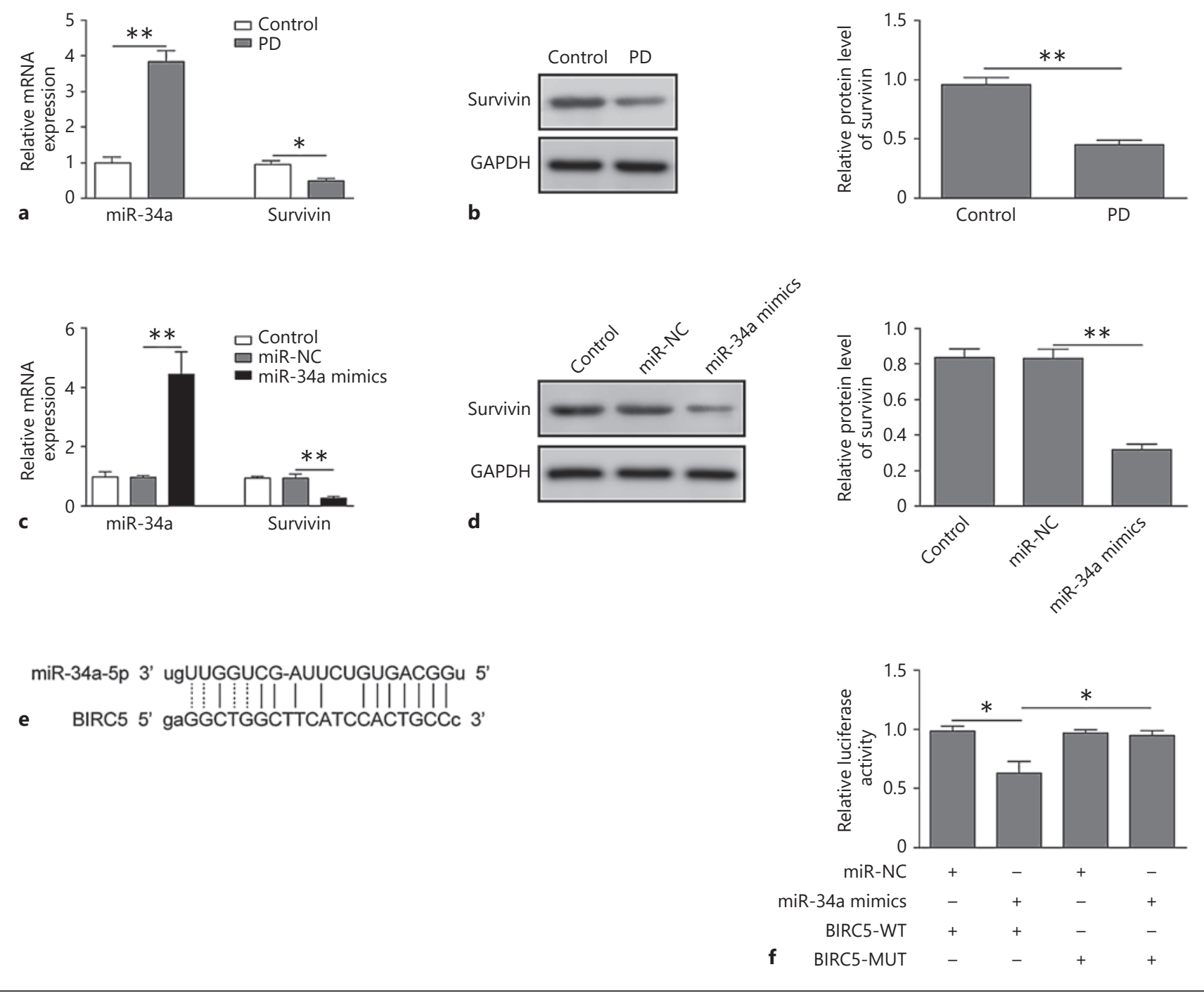

Fig. 3. miR-34a inhibited the expression of survivin. a qPCR analysis of the miR-34a and survivin level in SGC-7901 cells following treatment with PD $(10 \mu \mathrm{M})$ for $48 \mathrm{~h} .{ }^{*} p<0.05,{ }^{* *} p<0.01$ versus the control. $\mathbf{b}$ Western blot analysis of survivin in SGC-7901 cells after being treated with PD for $48 \mathrm{~h}$. The relative protein expression normalized to GAPDH. c, d The miR-34a and survivin expression levels were detected after SGC-7901 cells were transfect-

The miR-34a was overexpressed to discover the relationship between miR-34a and survivin. As shown in Figure $3 \mathrm{c}, \mathrm{d}$, miR-34a was significantly overexpressed after being transfected with miR-34a mimics, while survivin expression decreased both on mRNA and protein levels. These results showed that miR-34a overexpression significantly inhibited the expression of survivin. Based on the biological effects and expression levels of ed with the miR-34a mimics for $48 \mathrm{~h} .{ }^{* *} p<0.01$ versus miR-NC. e The miR-34a binding site within the $3^{\prime}$ UTR of human BIRC5 cDNA was predicted with RAID software. $\mathbf{f}$ Dual luciferase reporting assay was performed. Cells were cotransfected with the BIRC5WT vector or BIRC5-MUT vector and a plasmid containing miR34 a mimics or miR-NC. ${ }^{*} p<0.05$ versus miR-34a mimics+BIRC5WT.

miR-34a and survivin, we hypothesized that the transcript of BIRC5 may be a target of miR-34a. To further identify the relationship between miR-34a and survivin, RAID software was applied to assess the binding site of miR-34a and the transcript of BIRC5. As shown in Figure $3 \mathrm{e}$, bioinformatics analysis predicted that the $3^{\prime}$ UTR of the transcript of BIRC5 might bind to miR-34a. We then performed luciferase reporter assay to detect 

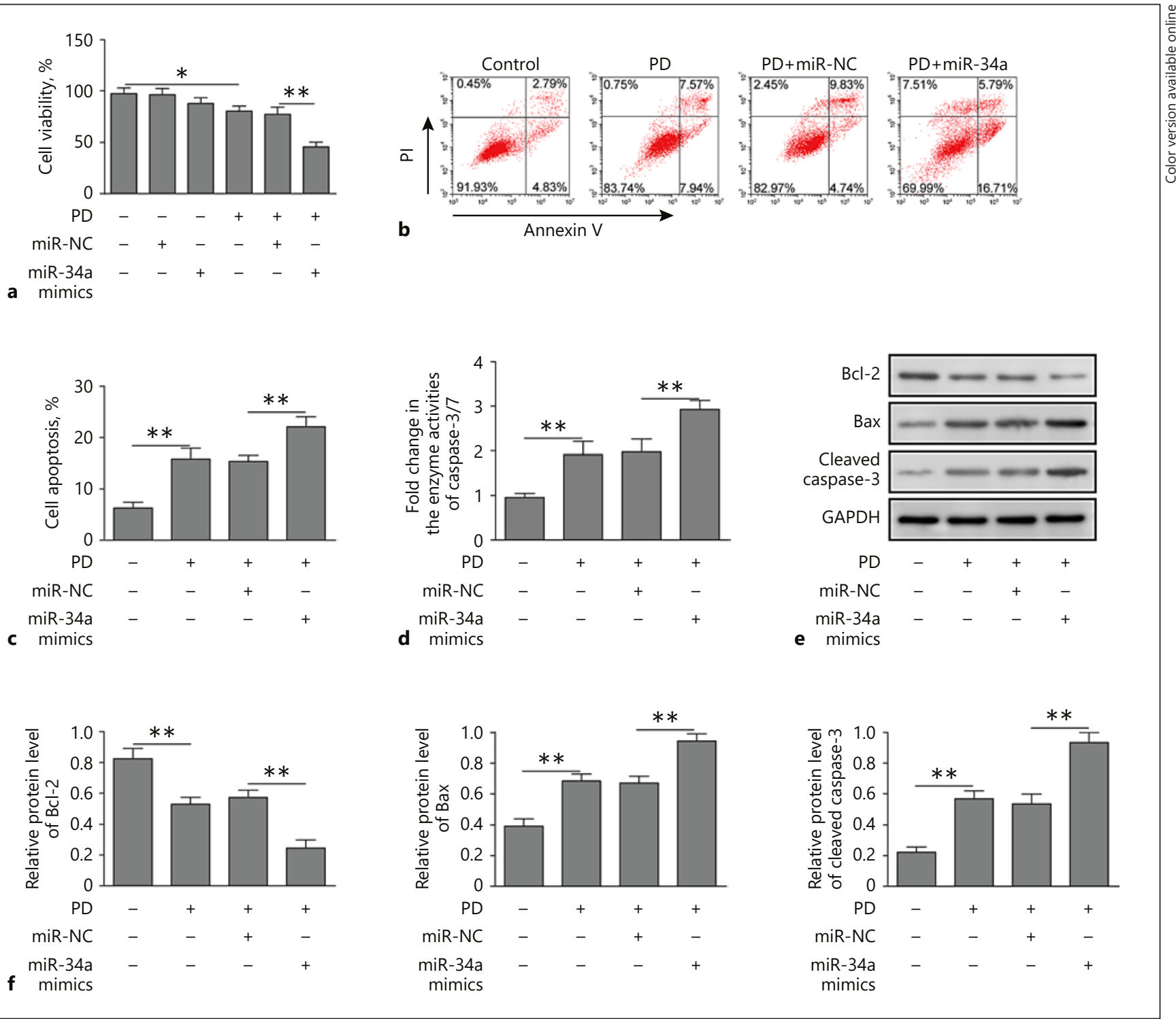

Fig. 4. miR-34a mimics could enhance the chemosensitivity of GC cells to PD. a Analysis of the cell growth rate by modified MTT assay with the treatment of PD, miR-34a mimics/miR-NC, PD, and miR-34a mimics combination, or PD and miR-NC combination. ${ }^{*} p<0.05$ versus the control or miR-NC; ${ }^{* *} p<0.01$ versus $\mathrm{PD}+$ miR-NC. b, c Representative apoptosis profiles obtained by FACS analysis from Annexin V-FITC and PI stained in treated SGC7901 cells. ${ }^{* *} p<0.01$ versus the control; ${ }^{* *} p<0.01$ versus $P D+m i R-$

whether miR-34a targets the transcript of BIRC5. As shown in Figure 3f, overexpression of miR-34a suppressed the luciferase activity of the BIRC5-WT group compared to the BIRC5-MUT group. These results support that miR-34a has a binding site with $3^{\prime}$-UTR of the transcript of BIRC5 and negatively regulated survivin expression in GC cells.

miR-34a Enhances the Susceptibility of GC Cells to PD
NC. d Caspase-3/7 activity was measured using a Caspase-Glo ${ }^{\circledR} 3 / 7$ detection kit. ${ }^{* *} p<0.01$ versus the control; ${ }^{* *} p<0.01$ versus PD+miR-NC. e Whole-cell lysates collected $48 \mathrm{~h}$ after SGC-7901 cell treatment were assessed by Western blot analysis using the antibodies of Bcl-2, Bax, and cleaved caspase-3. GAPDH was used as a loading control. ${ }^{* *} p<0.01$ versus the control; ${ }^{* *} p<0.01$ versus PD+miR-NC.

\section{Overexpression of miR-34a Enhances the Drug \\ Sensitivity of GC Cells to PD}

To detect the potential role of miR-34a in GC drug sensitivity to $\mathrm{PD}$, we transfected miR-34a mimics to SGC-7901 cells. As shown in Figure 4a, compared with the PD group, the proliferation of SGC-7901 decreased in the PD+miR-34a mimics group. Furthermore, flow 


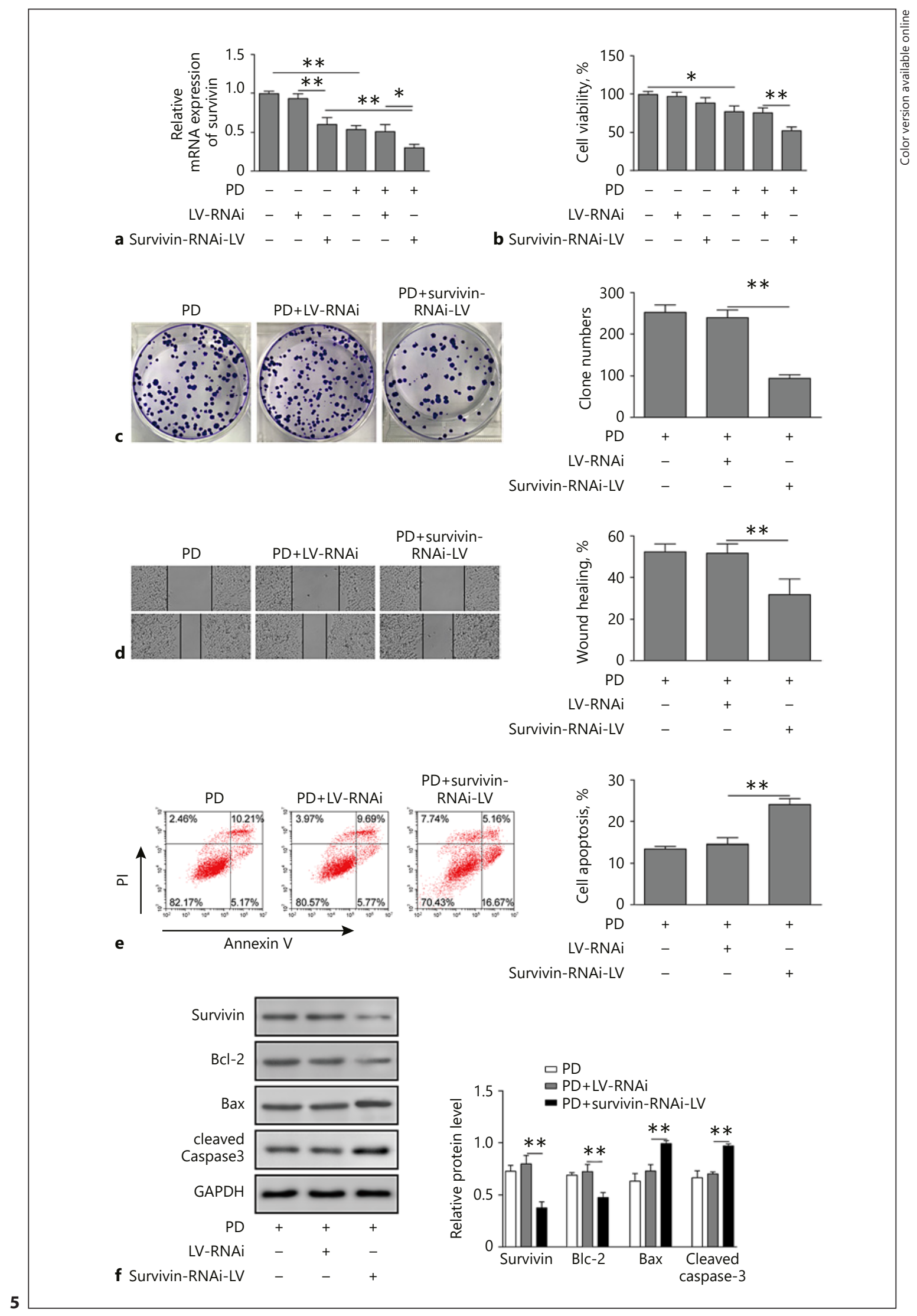

(For legend see next page.) 
cytometry analysis results indicated that combined with miR-34a, PD promoted the SGC-7901 apoptosis (Fig. 4b, c). In addition, we investigated that PD induced caspase-3/7 activity in SGC-7901 cells, which more remarkably increased in the PD+miR-34a mimics group (Fig. 4d).

Additionally, we analyzed the expression of several apoptotic-related proteins, Bcl-2, Bax, and cleaved caspase-3. Western blotting suggested that PD suppressed Bcl-2 expression, while it promoted the expression of Bax and cleaved caspase- 3 , which were more significant in the
PD+miR-34a mimics group (Fig. 4e). These results suggested that miR-34a acts as a tumor suppressor in GC cells and enhances the drug sensitivity of GC cells to PD.

\section{Downregulation of Survivin Enhances Drug Sensitivity} of GC Cells to PD

To investigate the potential mechanisms of survivin in the sensitivity of GC cells to PD, we treated SGC-7901 cells with $\mathrm{PD}$ alone, PD combined with RNAi-LV, or survivin-RNAi-LV. As shown in Figure $5 \mathrm{a}$ and b, the survivin expression level and cell viability were remarkably

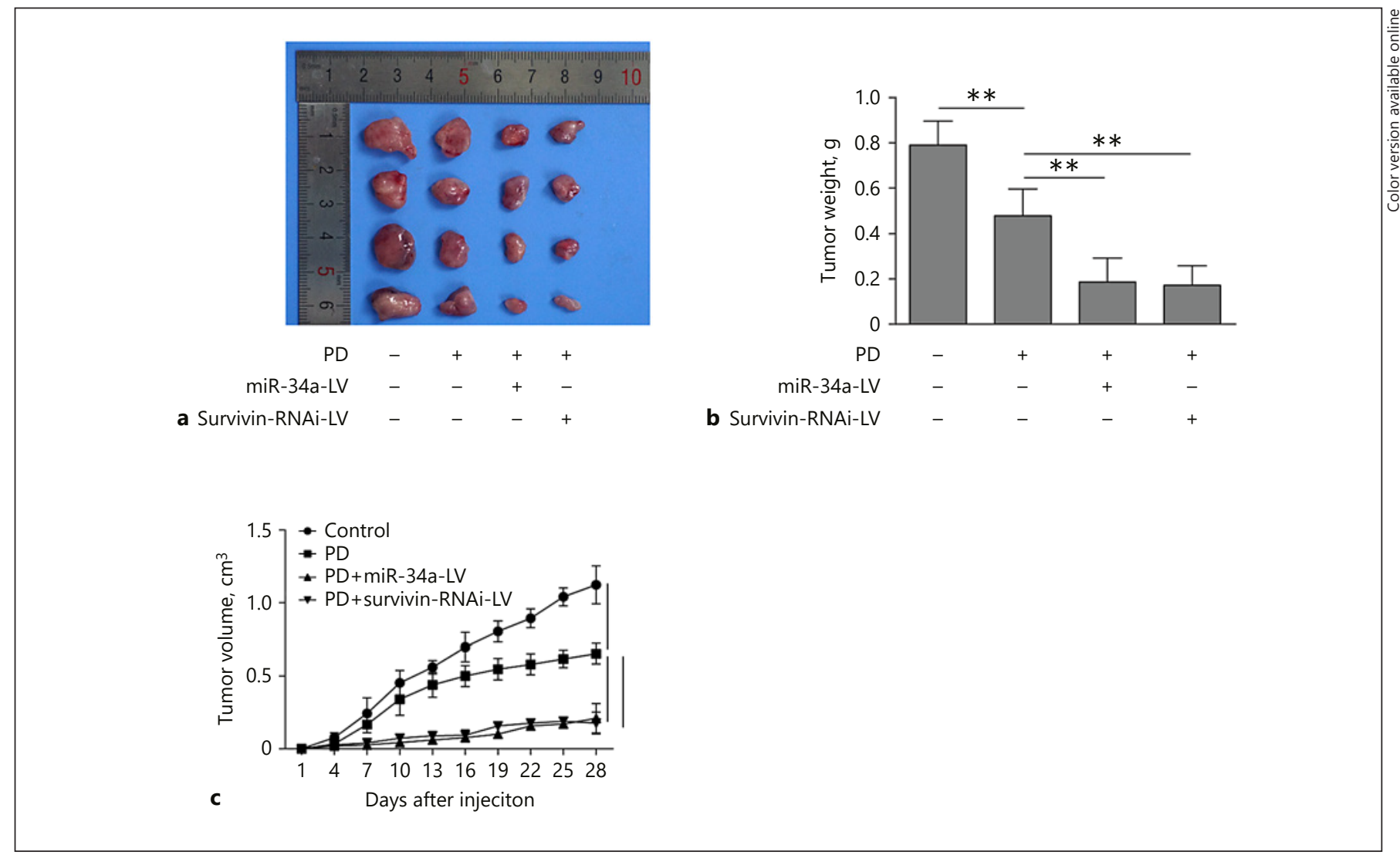

Fig. 6. miR-34a/survivin enhances the anti-tumor effect of PD on GC. a, b After sacrificing the mice, tumors were collected and weighed. ${ }^{* *} p<0.01$ versus the control; ${ }^{* *} p<0.01$ versus PD. c Tumor volumes were measured every 3 days for 28 days.

Fig. 5. Downregulation of survivin enhances the chemosensitivity of GC cells to PD. a The expression level of survivin was assessed by qPCR in GC cells with the treatment of PD, survivin-RNAi-LV/LVRNAi, PD and survivin-RNAi-LV combination, or PD and LVRNAi combination. ${ }^{*} p<0.05$ versus $\mathrm{PD}+\mathrm{LV}-\mathrm{RNAi}$; ${ }^{*} p<0.01$ versus the control or LV-RNAi or survivin-RNAi-LV. $\mathbf{b}$ Analysis of cell viability by modified MTT assay. ${ }^{*} p<0.05$ versus the control or LV-RNAi; ${ }^{* *} p<0.01$ versus PD+LV-RNAi. c Colony formation

miR-34a Enhances the Susceptibility of GC Cells to PD assay of SGC-7901 cell lines upon PD alone, PD, and LV-RNAi combination, or PD and survivin-RNAi-LV combination treatment. ** $p<0.01$ versus PD. d The migration of SGC-7901 cells was tested by wound healing assay. ${ }^{* *} p<0.01$ versus PD. e After being treated, cells were stained with Annexin V/PI and analyzed by flow cytometry. ${ }^{* *} p<0.01$ versus PD. $\mathbf{f}$ Western blot analysis on the level of survivin, Bcl-2, Bax, and cleaved caspase-3 expression in SGC-7901 cells. GAPDH was used as the internal control. ${ }^{* *} p<0.01$ versus PD. 
suppressed in GC cells treated with PD and/or survivinRNAi-LV. Soft agar colony formation and wound healing assay results showed that the proliferation and invasive abilities of SGC-7901 cells were evidently decreased in the $\mathrm{PD}+$ survivin-RNAi-LV group in comparison with the absence of survivin (Fig. $5 c$, d). Consistent with these data, the combination with survivin-RNAi-LV and PD promoted the SGC-7901 apoptosis (Fig. 5e).

Western blotting results indicated that the expression of survivin and $\mathrm{Bcl}-2$ decreased in the $\mathrm{PD}+$ survivinRNAi-LV group compared with PD+LV-RNAi, while the expression of Bax and cleaved caspase- 3 increased visibly in the PD+survivin-RNAi-LV group (Fig. 5f).

\section{miR-34a/Survivin Enhances the Anti-Tumor Effect of} $P D$ on $G C$

For further confirmation of the influence of miR-34a overexpression and survivin suppression on the anti-tumor effects of PD in vivo, we performed xenograft experiments with nude mice. After being sacrificed, the mice tumor tissues in the PD and miR-34a-LV or survivin-RNAi-LV cotreatment groups were evidently smaller than those in the PD group (Fig. 6a, b). After PD treatment, we found upregulation of miR-34a or inhibition of survivin markedly reduced the growth speed of the GC tumor (Fig. 6c). Collectively, miR-34a/survivin enhances the anti-tumor effect of PD on GC.

\section{Discussion}

GC remains one of the most prevalent malignant tumors in the world and manifests a high mortality. The application of herb ingredients is a hot issue in anti-tumor research, which has made great progress. It has been reported that ginsenoside Rg3 suppressed the development of colorectal cancer via targeting tumor angiogenesis and cancer stem cells [19]. In addition, honokiol, a phytochemical from Magnolia grandiflora, has been proven to inhibit oral carcinogenesis proliferation [20]. PD has been reported to have many pharmacological activities, including promoting apoptosis in GC cells [9]. In this study, we also demonstrated that PD suppressed the proliferation and induced the apoptosis in GC cells. miR-34a has been proven to act as a tumor suppressor in multiple malignant tumors, and is closely related to survivin [21]. The current study found that miR34 a was decreased in GC cells, while survivin was increased in comparison with the normal gastric epithelial cell line. The aim of our research was to inves- tigate the functions of miR-34a in enhancing the susceptibility of GC to PD.

miRNAs regulate gene expression in a posttranscriptional manner by targeting $3^{\prime} U T R s$ of mRNAs. In recent decades, miRNAs were found to take part in a variety of cellular processes, such as metabolic pathways, cell proliferation, apoptosis, and autophagy, etc. [22]. In malignant tumors, miRNAs are also involved into the regulation of multiple cellular behaviors, including invasion, migration, and drug resistance [10]. Multiple previous studies have proven the downregulation of miR-34a in a series of malignancies, including glioma [23], lung adenocarcinoma [24], and hepatocellular carcinoma [25]. Zhang et al. [26] reported that miR-34a could sensitize colorectal cancer cells to 5-fluorouracil. Zhou et al. [15] also proposed that miR-34a could promote the susceptibility of GC cells to luteolin, a natural anti-tumor flavonoid separated from herbs. In our research, miR-34a was found to be downregulated in GC, and its upregulation obviously enhanced the tumor-suppressive effects of PD, indicating that miR-34a could be a candidate for improving the drug susceptibility in a variety of drug therapies.

Survivin, a typical apoptotic suppressive protein, has been demonstrated to regulate caspase activity. Accumulating evidence supports that survivin is overexpressed and contributes to the resistance to chemotherapeutic drugs in multiple malignancies [27-29]. Moreover, survivin also protects tumor angiogenesis through preventing vascular endothelial cells from apoptosis [30]. Therefore, survivin is currently considered as an ideal therapeutic target. In our present study, PD induced upregulation of miR-34a and downregulation of survivin. Moreover, overexpression of miR-34a could directly inhibit survivin expression. Thus, PD may induce expression of miR-34a and consequently reduce survivin expression. However, there are many miRNAs that can bind to 3'UTR of survivin and suppress its level in GC cells. Nor do we rule out that PD downregulates survivin by affecting the expression of other miRNAs. Knockdown of survivin significantly enhanced the tumor suppressive effects of PD in GC cells, which is similar to the phenomenon observed after miR-34a mimics transfection. Also, in vivo results showed that treatment of miR$34 \mathrm{a}$ overexpression or survivin suppression and PD could more significantly inhibit tumor growth than PD alone. Together, it is indicated that the drug-sensitizing effects of miR-34a may be established by the inhibition of survivin.

In conclusion, our study has shown that miR-34a can enhance the sensitivity of GC to PD via regulating the ex- 
pression of survivin, which leads to suppression of the GC tumor growth. These results support that the combination of miR-34a upregulation and PD may provide a promising therapeutic for the treatment of GC.

\section{Acknowledgements}

This work was supported by the study of survivin-RNA gene silence enhancing the sensitivity of gastric cancer cells to traditional Chinese medicine Sanwubai powder (No. 81503561).

\section{Statement of Ethics}

The animal care and experimental protocols were approved by the Institutional Animal Care and Use Committee of Hunan University of Traditional Chinese Medicine.

\section{Disclosure Statement}

The authors have no conflicts of interest to declare.

\section{References}

1 Karimi P, Islami F, Anandasabapathy S, Freedman ND, Kamangar F. Gastric cancer: descriptive epidemiology, risk factors, screening, and prevention. Cancer Epidemiol Biomarkers Prev. 2014 May;23(5):700-13.

2 Song Z, Wu Y, Yang J, Yang D, Fang X Progress in the treatment of advanced gastric cancer. Tumour Biol. 2017 Jul;39(7): 1010428317714626

3 ZiaSarabi P. Sorayayi S, Hesari A, Ghasemi F. Circulating microRNA-133, microRNA-17 and microRNA-25 in serum and its potential diagnostic value in gastric cancer. J Cell Biochem. 2019;120(8):12376-81.

4 Zhang Z, Zhao M, Zheng W, Liu Y. Platycodin D, a triterpenoid saponin from Platycodon grandiflorum, suppresses the growth and invasion of human oral squamous cell carcinoma cells via the NF- $\kappa B$ pathway. J Biochem Mol Toxicol. 2017 Sep;31(9):e21934.

5 Ye Y, Pei L, Ding J, Wu C, Sun C, Liu S. Effects of Platycodin D on S100A8/A9-induced inflammatory response in murine mammary carcinoma 4T1 cells. Int Immunopharmacol. 2019 Feb; 67:239-47.

6 Kong Y, Lu ZL, Wang JJ, Zhou R, Guo J, Liu J, et al. Platycodin D, a metabolite of Platycodin grandiflorum, inhibits highly metastatic MDA-MB-231 breast cancer growth in vitro and in vivo by targeting the MDM2 oncogene. Oncol Rep. 2016 Sep;36(3):1447-56.

$7 \mathrm{Li} \mathrm{T}$, Xu XH, Tang ZH, Wang YF, Leung CH, Ma DL, et al. Platycodin D induces apoptosis and triggers ERK- and JNK-mediated autophagy in human hepatocellular carcinoma BEL-7402 cells. Acta Pharmacol Sin. 2015 Dec;36(12):1503-13.

8 Kim HL, Park J, Jung Y, Ahn KS, Um JY. Platycodin D, a novel activator of AMP-activated protein kinase, attenuates obesity in $\mathrm{db} / \mathrm{db}$ mice via regulation of adipogenesis and thermogenesis. Phytomedicine. 2019 Jan;52:254-63.

9 Chun J, Joo EJ, Kang M, Kim YS. Platycodin $\mathrm{D}$ induces anoikis and caspase-mediated apoptosis via $\mathrm{p} 38$ MAPK in AGS human gastric cancer cells. J Cell Biochem. 2013 Feb; 114(2):456-70.

10 Rupaimoole R, Slack FJ. MicroRNA therapeutics: towards a new era for the manage- ment of cancer and other diseases. Nat Rev Drug Discov. 2017 Mar;16(3):203-22.

11 Yu X, Gao Y, Zhang F. Propofol inhibits pancreatic cancer proliferation and metastasis by upregulating miR-328 and downregulating ADAM8. Basic Clin Pharmacol Toxicol. 2019;125(3):271-8.

12 Zhou R, Zhang Y, Du G, Han L, Zheng S, Liang $\mathrm{J}$, et al. Down-regulated let-7b-5p represses glycolysis metabolism by targeting AURKB in asthenozoospermia. Gene. 2018 Jul;663:83-7.

$13 \mathrm{Li}$ Z, Liu ZM, Xu BH. A meta-analysis of the effect of microRNA-34a on the progression and prognosis of gastric cancer. Eur Rev Med Pharmacol Sci. 2018 Dec;22(23):8281-7.

14 Ping W, Senyan H, Li G, Yan C, Long L. Increased Lactate in Gastric Cancer Tumor-Infiltrating Lymphocytes Is Related to Impaired T Cell Function Due to miR-34a Deregulated Lactate Dehydrogenase A. Cell Physiol Biochem. 2018;49(2):828-36.

15 Zhou Y, Ding BZ, Lin YP, Wang HB. MiR$34 \mathrm{a}$, as a suppressor, enhance the susceptibility of gastric cancer cell to luteolin by directly targeting HK1. Gene. 2018 Feb;644:56-65.

16 Reichert S, Rödel C, Mirsch J, Harter PN, Tomicic MT, Mittelbronn M, et al. Survivin inhibition and DNA double-strand break repair: a molecular mechanism to overcome radioresistance in glioblastoma. Radiother Oncol. 2011 Oct;101(1):51-8.

17 Werner TA, Dizdar L, Nolten I, Riemer JC, Mersch S, Schütte SC, et al. Survivin and XIAP - two potential biological targets in follicular thyroid carcinoma. Sci Rep. 2017 Sep; $7(1): 11383$.

18 Guo L, Huang C, Ji QJ. Aberrant promoter hypermethylation of p16, survivin, and retinoblastoma in gastric cancer. Bratisl Lek Listy. 2017;118(3):164-8.

19 Tang YC, Zhang Y, Zhou J, Zhi Q, Wu MY, Gong FR, et al. Ginsenoside Rg3 targets cancer stem cells and tumor angiogenesis to inhibit colorectal cancer progression in vivo. Int J Oncol. 2018 Jan;52(1):127-38.

20 Eastham LL, Howard CM, Balachandran P, Pasco DS, Claudio PP. Eating green: shining light on the use of dietary phytochemicals as a modern approach in the prevention and treatment of head and neck cancers. Curr Top Med Chem. 2018;18(3):182-91.

21 Liu Y, Zhang X, Chen J, Li T. Inhibition of mircoRNA-34a enhances survival of human bone marrow mesenchymal stromal/stem cells under oxidative stress. Med Sci Monit. 2018 Jan;24:264-71.

22 Gambari R, Brognara E, Spandidos DA, Fabbri E. Targeting oncomiRNAs and mimicking tumor suppressor miRNAs: vew trends in the development of miRNA therapeutic strategies in oncology (Review) [Review]. Int J Oncol. 2016 Jul;49(1):5-32.

23 Ma S, Fu T, Zhao S, Gao M. MicroRNA-34a-5p suppresses tumorigenesis and progression of glioma and potentiates Temozolomide-induced cytotoxicity for glioma cells by targeting HMGA2. Eur J Pharmacol. 2019 Jun;852:42-50.

24 Kim JS, Kim EJ, Lee S, Tan X, Liu X, Park S, et al. MiR-34a and miR-34b/c have distinct effects on the suppression of lung adenocarcinomas. Exp Mol Med. 2019 Jan;51(1):9.

25 Wu G, Li Z, Wang Y, Ju X, Huang R. miR-34a inhibits cell proliferation by targeting SATB2 in hepatocellular carcinoma. BioMed Res Int. 2018 Dec;2018:2863902.

26 Zhang Q, Wang J, Li N, Liu Z, Chen Z, Li Z, et al. miR-34a increases the sensitivity of colorectal cancer cells to 5 -fluorouracil in vitro and in vivo. Am J Cancer Res. 2018 Feb; 8(2):280-90.

27 Wang S, Xu Y, Chan HF, Kim HW, Wang Y, Leong KW, et al. Nanoparticle-mediated inhibition of survivin to overcome drug resistance in cancer therapy. J Control Release. 2016 Oct;240:454-64.

28 Zhou C, Zhu Y, Lu B, Zhao W, Zhao X. Survivin expression modulates the sensitivity of A549 lung cancer cells resistance to vincristine. Oncol Lett. 2018 Oct;16(4):5466-72.

29 Chawla D, Kar R, Gupta B, Halder S, Garg S, Mehndiratta M, et al. Role of Survivin and p53 Expression in Response of Primary Culture of Ovarian Cancer Cells to Treatment With Chemotherapeutic Agents. Int J Gynecol Cancer. 2018 Jul;28(6):1239-46.

30 Altieri DC. The molecular basis and potential role of survivin in cancer diagnosis and therapy. Trends Mol Med. 2001 Dec;7(12):542-7. 